\title{
Occurrence of co-infection with dengue viruses during 2014 in New Delhi, India
}

\author{
A. TAZEEN ${ }^{1}$, N. AFREEN ${ }^{1}$, M. ABDULLAH ${ }^{2}$, F. DEEBA ${ }^{1}$, S. H. HAIDER ${ }^{1}$, \\ S. N. KAZIM ${ }^{1}$, S. ALI ${ }^{1}$, I. H. NAQVI ${ }^{3}$, S. BROOR ${ }^{4}, A \cdot$ AHMED $^{5}$ AND \\ S. PARVEEN ${ }^{1 *}$ \\ ${ }^{1}$ Centre for Interdisciplinary Research in Basic Sciences, Jamia Millia Islamia, New Delhi, India \\ ${ }^{2}$ School of Life Sciences, Jaipur National University, Jaipur, India \\ ${ }^{3}$ Dr. M. A. Ansari Health Centre, Jamia Millia Islamia, New Delhi, India \\ ${ }^{4}$ Department of Microbiology, Faculty of Medicine and Health Science, Shree Guru Gobind Singh Tricentenary \\ University, Gurgaon, Haryana, India \\ ${ }^{5}$ Protein Research Chair, Department of Biochemistry, College of Science, King Saud University, Riyadh, Saudi \\ Arabia
}

Received 17 December 2015; Final revision 9 June 2016; Accepted 9 August 2016; first published online 13 September 2016

\section{SUMMARY}

Dengue fever is an arthropod-borne viral infection that has become endemic in several parts of India including Delhi. We studied occurrence of co-infection with dengue viruses during an outbreak in New Delhi, India in 2014. For the present study, blood samples collected from symptomatic patients were analysed by RT-PCR. Eighty percent of the samples were positive for dengue virus. The result showed that DENV-1 (77\%) was the predominant serotype followed by DENV-2 (60\%). Concurrent infection with more than one serotype was identified in $43 \%$ of the positive samples. Phylogenetic analysis clustered DENV-1 strains with the American African and DENV-2 strains in Cosmopolitan genotypes. Four common amino-acid mutations were identified in the envelope gene of DENV-1 sequences (F337I, A369T, V380I and L402F) and one common mutation (N390S) in the DENV-2 sequences. Further analysis revealed purifying selection in both the serotypes. A significant number of patients were co-infected with DENV-1 and DENV-2 serotypes. Although we do not have direct evidence to demonstrate co-evolution of these two stereotypes, nonetheless their simultaneous occurrence does indicate that they are favoured by evolutionary forces. An ongoing surveillance and careful analysis of future outbreaks will strengthen the concept of co-evolution or otherwise. Whether the concurrent dengue viral infection is correlated with disease severity in a given population is another aspect to be pursued. This study is envisaged to be useful for future reference in the context of overall epidemiology.

Key words: Concurrent infections, dengue virus, phylogenetic analysis, selection pressure analysis.

\section{INTRODUCTION}

Dengue fever is an emerging acute mosquito-borne viral disease resulting in a great socioeconomic

\footnotetext{
* Author for correspondence: Dr S. Parveen, Centre for Interdisciplinary Research in Basic Sciences, Jamia Millia Islamia, New Delhi, India.

(Email: sparveen2@jmi.ac.in; shamp25@yahoo.com)
}

burden. It is prevalent in several tropical and subtropical regions of the world. The causative agent of the fever is dengue virus (DENV), which belongs to family Flaviviridae, genus Flavivirus. There are four (DENV-1 to DENV-4) different antigenically distinct serotypes of dengue virus. Infection with one type of the serotype imparts lifelong immunity to that 
serotype. The four serotypes are further divided genotypically into 4-6 genotypes.

Recent studies have reported co-circulation of multiple serotypes in different geographical regions of the world including India [1-3]. In addition, concurrent infections with more than one serotype of dengue virus have been documented in various studies [2, 46]. However, the true disease burden or collective severity due to dual infection remains unknown. Moreover, the information on correlation of disease severity with the serotypes or genotypes is also limited. Finally, there seems to be lack of information on the range of titre of a given serotype in different ethnic groups from different parts of the world. Information on these areas would provide a new dimension towards understanding the susceptibility or proneness of a population and possible triggering mechanism of the infection in the context of varying genotypes.

The present study was planned to detect dengue viruses by reverse transcriptase-polymerase chain reaction (RT-PCR) in blood samples collected from suspected patients during an outbreak in New Delhi in 2014. The concurrent infections were identified by RT-PCR. Further, the molecular characterization of dengue viruses was performed by DNA sequencing followed by phylogenetic analysis. The data provide information on the circulating dengue virus strains from New Delhi, India delineating evolutionary trajectory of the prevalent strain compared to other strains. The present study is envisaged to help in correlating outbreaks with various causative factors enabling effective control measures.

\section{MATERIAL AND METHODS}

\section{Collection of clinical samples}

The blood samples were collected from patients suspected of dengue virus infection attending the outpatient department of Dr. M. A. Ansari Health Centre, Jamia Millia Islamia, New Delhi. The health centre provides basic medical facilities to the 60000 university students, employees and their dependants. Background information (age, sex, clinical symptoms) was collected in the profomas from the patients. Informed consent was obtained from each patient in English or Hindi. The Institutional Ethics Committee of Jamia Millia Islamia approved the study. Trained medical professionals collected about $2-3 \mathrm{ml}$ of blood from each patient. The blood samples were transported to the virology laboratory within 2-3 h. Sera was separated from the samples and stored at $-70{ }^{\circ} \mathrm{C}$.

\section{RNA extraction and cDNA synthesis}

The RNA was extracted from $140 \mu$ l serum samples using QIAamp Viral RNA extraction kit (Qiagen, Germany) according to the manufacturer's instructions. RNA was eluted in $50 \mu$ l elution buffer and stored at $-70{ }^{\circ} \mathrm{C}$. The cDNA was synthesized from the extracted RNA using AMV-RT and random hexamer as described previously [7].

\section{Detection of dengue virus by PCR typing}

Dengue virus was detected in the clinical samples by a nested PCR reaction using published primers and cycling conditions [8]. The CprM region of the dengue virus genome was the target for amplification. The nested PCR was performed as described in our previous publication [7]. Briefly the cDNA amplification was performed in $25 \mu \mathrm{l}$ reaction volume, with Taq DNA polymerase (Genei, Bangalore), dNTPs (Promega, USA), forward (D1) and reverse (D2) primers [8] in a thermal cycler (Applied Biosystems, USA). In the second round of seminested PCR, the external amplicon was amplified using published primers. Then $1 \mu$ diluted amplicon was reamplified with serotype-specific primers for DENV-1, DENV-2, DENV-3 and DENV-4, respectively. The nested PCR reaction was set up with primers (TS1, TS2, TS3, TS4 and D1) [8], dNTPs (Promega, USA), Taq polymerase (Genei, India), in a $25 \mu 1$ reaction volume. The amplicons were resolved on $2 \%$ agarose gel by electrophoresis and were visualized under UV light on a gel documentation system (Wealtec, USA).

\section{Sequencing PCR}

Partial segments of domain III of the envelope gene of DENV-1 and DENV-2 was amplified with the published primers $[9,10]$. An aliquot of $3 \mu \mathrm{l}$ cDNA was amplified in a $25 \mu 1$ reaction with forward primer, reverse primer, Taq DNA polymerase (Genei, India) and dNTPs (Promega, USA). Envelope protein gene regions of two different lengths, $448 \mathrm{bp}$ and $574 \mathrm{bp}$, were amplified for DENV-1 and DENV-2, respectively. These two PCR reactions were performed as 
described previously [7]. The required bands were excised from agarose gel. The DNA was extracted from gel using the QIAquick Gel Extraction kit (Qiagen, Germany) according to the manufacturer's instructions.

\section{DNA sequencing and sequence analysis}

Partial nucleotide sequences of the $\mathrm{E}$ protein gene of DENV-1 and DENV-2 were determined by commercial sequencing (Applied Biosystem, USA). The amplicons were sequenced in both forward and reverse directions. The sequences were confirmed by BLAST (http://blast.ncbi.nlm.nih.gov/Blast.cgi). The sequences were manually aligned and edited to resolve nucleotide ambiguities and to obtain consensus sequence using GeneDoc v. 2.7 (http://genedoc. software.informer.com/2.7/) and BioEdit v. 7.2 (http:// bioedit.software.informer.com/7.2/). Multiple sequence alignment was conducted using CLUSTAL X2 (http://www.clustal.org/clustal2/).

\section{Phylogenetic analysis}

Phylogenetic trees were constructed by the maximumlikelihood method using MEGA 6.06 software (http:// mega6.software.informer.com/). Genetic distances were calculated using the Tamura-Nei model of nucleotide substitution. The robustness of the tree was assessed with 1000 bootstrap replicates. The prototype strain used for DENV-1 was the Hawaii strain (GenBank accession no. EU848545) and Thailand strain, 16681 (GenBank accession no. U87411) for DENV-2.

\section{Selection pressure analysis}

Selection pressure was studied in the codons of the envelope protein gene using a Datamonkey web-server (http://www.datamonkey.org/). The non-synonymous to synonymous mutations ratio $(\mathrm{dN} / \mathrm{dS})$ was estimated using three different approaches, including single likelihood ancestor counting (SLAC), fixed effects likelihood (FEL) and random effects likelihood (REL) by using the HKY85 and F81 models of nucleotide substitution. The positively selected sites were defined as the sites that were under positive selection by at least two different methods with $P \leqslant 0 \cdot 1$ for SLAC and FEL and Bayes factor $\geqslant 50$ for REL.

\section{Ethical standards}

The authors assert that all procedures contributing to this work comply with the ethical standards of the relevant national and institutional committees on human experimentation and with the Helsinki Declaration of 1975, as revised in 2008.

\section{RESULTS}

\section{Patients' characteristics}

The symptomatic patients were recruited from the outpatient department of Dr. M. A. Ansari Health Centre, Jamia Millia Islamia over a period of 3 months from September to November 2014 during an outbreak in Delhi. A total of 60 blood samples were collected from suspected patients during the study. Supplementary Table S1 shows the details of patients enrolled for the current study with their age, gender, platelet count and number of days of fever. The mean age $( \pm$ s.D.) of patients was $26.71( \pm 9 \cdot 63)$ years. Mean days of illness was $4.91( \pm 2.61)$ days. The male:female ratio was $2 \cdot 52: 1$.

\section{Characteristics of co-infected vs. mono-infected patients}

The samples collected from co-infected patients during the outbreak showed clinical symptoms of dengue viral infection like fever, headache, nausea, bodyache, weakness, etc. Fever was the most common symptom observed in all co-infected patients. The duration of fever ranged from 1 to 6 days. The mean age $( \pm$ s.D.) of the co-infected patients was 20.04 ( $\pm 12 \cdot 36)$ years. Mean days of illness in co-infected patients was $3.80( \pm 1.72)$ days. The platelet count of the coinfected patients ranged from 0.6 to $3.5 \mathrm{lac} / \mathrm{mm}^{3}$. Three patients showed severe disease with mild haemorrhagic tendencies. One patient with severe disease (patient ID 70/14) had a platelet count of $1.8 \mathrm{lac}$ $\mathrm{mm}^{3}$ with 7 days of fever. The second patient (patient ID 73/14) had a platelet count of $1.8 \mathrm{lac} / \mathrm{mm}^{3}$ with 3 days of fever. The third patient was aged 15 years and had a low platelet count of $0.6 \mathrm{lac} / \mathrm{mm}^{3}$ with 4 days of fever. At the same time the mono-infected patients also showed similar clinical symptoms with fever being the most common symptom. The platelet count of mono-infected patients ranged from 1.8 to $3.7 \mathrm{lac} / \mathrm{mm}^{3}$. None of the mono-infected patient had haemorrhagic tendencies. 


\section{RT-PCR}

All the samples collected during the outbreak were tested for dengue virus infection by RT-PCR. Forty-eight samples $(80 \%)$ were positive for dengue virus out of 60 samples tested by RT-PCR. Multiplex nested PCR detected two different serotypes, DENV-1 and DENV-2 in the positive samples. A $482 \mathrm{bp}$ and a $119 \mathrm{bp}$ band were visualized on the agarose gel for DENV-1 and DENV-2, respectively. The male:female ratio for the dengue virus-positive sample was $2 \cdot 69: 1$. Thirty-seven $(77 \%)$ samples were positive for DENV-1 and $29(60 \%)$ samples for DENV-2 including concurrent infections. Coinfection with more than one type of serotype was identified in $21(43 \%)$ of the positive samples.

\section{DNA sequencing}

The dengue virus-positive samples for both serotypes identified during the outbreak were amplified with another set of published primers for domain III of the envelope protein gene for DNA sequencing. A total of ten samples, six for DENV-2 and four for DENV-1 were sequenced in both forward and reverse direction. All four DENV-1 and six DENV-2 sequences were confirmed by BLAST. The sequences of DENV-1 strains were submitted to GenBank (accession nos. KT355376-KT355379). The sequences of DENV-2 strains were submitted to GenBank (accession nos. KT355380-KT355385).

\section{Phylogenetic analysis}

\section{Phylogenetic analysis of DENV-1}

The aligned region was 312 bp (104 amino acids) corresponding to 991-1302 bp of E gene and 1926-2237 $\mathrm{bp}$ of the full genome of the prototype strain. The study sequences showed a nucleotide distance of 9$10 \%$ and an amino-acid distance of $4-6 \%$ with respect to the prototype strain. The study sequences showed nucleotide distances of $1 \%$ and amino-acid distances of $2 \%$ between themselves. Seventy-four sequences (including four study sequences) of different genotypes of DENV-1 were downloaded from GenBank and used to construct the phylogenetic tree. These included 15 sequences from India (11 published sequences and four study sequences). The accession numbers of the sequences that were used for the present study are given in Supplementary Table S2a. The study sequences clustered with the American African genotype by phylogenetic analysis (Fig. 1).

The study sequences showed a total of 25 mutations at the nucleotide level in all the strains with respect to the prototype strain. One particular strain (DL/ DENV-1/125/14) showed additional four mutations. Four common amino-acid mutations (F337I, A369T, V380I and L402F) were identified in all the study sequences that were reported earlier. Another strain (DL/DENV-1/125/14) showed additional two aminoacid mutations besides the common ones (F429N and V432F) (Table 1a).

\section{Phylogenetic analysis of DENV-2}

The aligned region was $465 \mathrm{bp}$ (155 amino acids) corresponding to 871-1335 bp of E gene and 1807-2271 $\mathrm{bp}$ of the full genome of the prototype strain. The study sequences showed nucleotide distance of $6-8 \%$ and amino-acid distance of $1-3 \%$ with respect to the prototype strain. The study sequences showed nucleotide distances of $1 \%$ and amino-acid distances of $3 \%$ between themselves. Sequences of 73 DENV-2 strains (including six study sequences) of different genotypes were downloaded from GenBank and used to construct the tree in the present study. These included 18 sequences from India (12 published sequences and six study sequences). The accession numbers of the sequences used in the present study are given in Supplementary Table S2b. The study sequences clustered with Cosmopolitan genotype by phylogenetic analysis (Fig. 2).

The study sequences had a total of 28 mutations at the nucleotide level in all the strains with respect to the prototype strain. Three strains (DL/DENV-2/69/14, DL/DENV-2/85/14 and DL/DENV-2/89/14) showed some additional mutations also. One common amino-acid mutation (N390S) was identified in all the sequences. Other amino-acid mutations were identified at different sites in the study sequences. Two different strains (DL/DENV-2/69/14 and DL/DENV-2/ 89/14) showed a common mutation, i.e. T404I. One particular strain (DL/DENV-2/85/14) showed four different mutations, i.e. A419P, L425P, Y444H and G445W (Table 1b). All the mutations have been reported earlier.

\section{Selection pressure analysis}

The selection pressure analysis of DENV-1 strains revealed a low ratio of $\mathrm{dN} / \mathrm{dS}(0 \cdot 1-0 \cdot 5)$ by using 


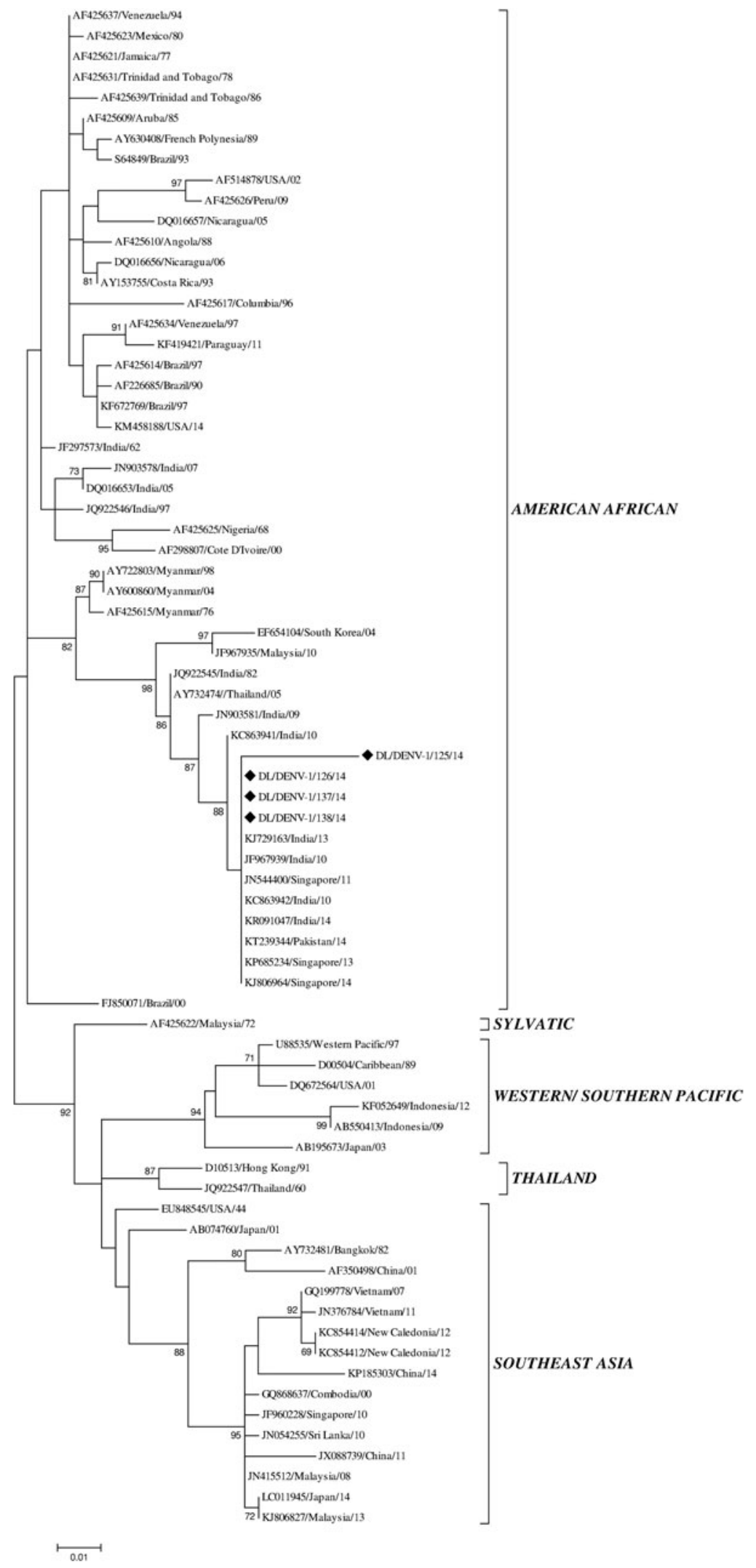

Fig. 1. Maximum likelihood phylogenetic tree of DENV-1 strains. The list of 74 nucleotide sequences that were used to construct the tree is given in Supplementary Table S2a. The study sequences are indicated by a diamond symbol ( $)$. Bootstrap values are represented by the numbers on nodes generated by 1000 replications. Values $>65 \%$ are shown in the tree. 
Table 1. Amino-acid substitutions in the envelope protein sequences for (a) DENV-1 and (b) DENV-2 serotypes. The mutations are shown in comparison to the respective prototype strains

\begin{tabular}{|c|c|c|c|c|c|c|}
\hline \multirow{2}{*}{$\frac{\text { (a) DENV-1 }}{\text { Hawaii strain }}$} & \multicolumn{6}{|c|}{ Substituted amino-acid position } \\
\hline & $337(\mathrm{~F})$ & $369(\mathrm{~A})$ & $380(\mathrm{~V})$ & $402(\mathrm{~L})$ & $429(\mathrm{~F})$ & $432(\mathrm{~V})$ \\
\hline DL/DENV-1/125/14 & $\mathrm{F} \rightarrow \mathrm{I}$ & $\mathrm{A} \rightarrow \mathrm{T}$ & $\mathrm{V} \rightarrow \mathrm{I}$ & $\mathrm{L} \rightarrow \mathrm{F}$ & $\mathrm{F} \rightarrow \mathrm{I}$ & $\mathrm{V} \rightarrow \mathrm{F}$ \\
\hline DL/DENV-1/126/14 & $\mathrm{F} \rightarrow \mathrm{I}$ & $\mathrm{A} \rightarrow \mathrm{T}$ & $\mathrm{V} \rightarrow \mathrm{I}$ & $\mathrm{L} \rightarrow \mathrm{F}$ & - & - \\
\hline DL/DENV-1/137/14 & $\mathrm{F} \rightarrow \mathrm{I}$ & $\mathrm{A} \rightarrow \mathrm{T}$ & $\mathrm{V} \rightarrow \mathrm{I}$ & $\mathrm{L} \rightarrow \mathrm{F}$ & - & - \\
\hline DL/DENV-1/138/14 & $\mathrm{F} \rightarrow \mathrm{I}$ & $\mathrm{A} \rightarrow \mathrm{T}$ & $\mathrm{V} \rightarrow \mathrm{I}$ & $\mathrm{L} \rightarrow \mathrm{F}$ & - & - \\
\hline (b) DENV-2 & \multicolumn{6}{|c|}{ Substituted amino-acid position } \\
\hline 16681 strain & $390(\mathrm{~N})$ & 404(T) & $419(\mathrm{~A})$ & $425(\mathrm{~L})$ & 444(I) & $445(\mathrm{~W})$ \\
\hline DL/DENV-2/69/14 & $\mathrm{N} \rightarrow \mathrm{S}$ & $\mathrm{T} \rightarrow \mathrm{I}$ & - & - & - & - \\
\hline DL/DENV-2/70/14 & $\mathrm{N} \rightarrow \mathrm{S}$ & - & - & - & - & - \\
\hline DL/DENV-2/74/14 & $\mathrm{N} \rightarrow \mathrm{S}$ & - & - & - & - & - \\
\hline DL/DENV-2/81/14 & $\mathrm{N} \rightarrow \mathrm{S}$ & - & - & - & - & - \\
\hline DL/DENV-2/85/14 & $\mathrm{N} \rightarrow \mathrm{S}$ & - & $\mathrm{A} \rightarrow \mathrm{P}$ & $\mathrm{L} \rightarrow \mathrm{P}$ & $\mathrm{I} \rightarrow \mathrm{H}$ & $\mathrm{W} \rightarrow \mathrm{H}$ \\
\hline DL/DENV-2/89/14 & $\mathrm{N} \rightarrow \mathrm{S}$ & $\mathrm{T} \rightarrow \mathrm{I}$ & - & - & - & - \\
\hline
\end{tabular}

different methods suggesting that the codon positions are relatively conserved (Table $2 a$ ). The first dataset, consisting of DENV-1 sequences of all the genotypes $(n=74)$, gave a $\mathrm{dN} / \mathrm{dS}$ ratio of $0 \cdot 105$ by the SLAC method using the HKY85 method of nucleotide substitution. SLAC analysis further showed no positive selection in this dataset, but 20 negatively selected sites were observed (data not shown). FEL analysis revealed 42 negatively selected sites and one weak positively selected site (codon number 98), 36 negatively selected sites and two weak positively selected sites (codon numbers 56 and 98) were observed by REL analysis. The F81 nucleotide model of substitution also revealed two (codon numbers 9 and 98) and three weak positively selected sites $(9,56$ and 98), respectively, by FEL and REL analysis. Another dataset comprising only the sequences of American African genotype $(n=49)$ also showed low $\mathrm{dN} / \mathrm{dS}$ ratio $(0.352)$ by SLAC analysis using the HKY85 method of nucleotide substitution. Further, this dataset showed seven and one negatively selected sites by SLAC and FEL, respectively. The F81 method of nucleotide substitution revealed two weak positively selected sites (codon numbers 9 and 98) by FEL analysis. One particular codon at position 98 was found to be positively selected by both FEL and REL analysis using two different methods of nucleotide substitution in both the datasets.

The DENV-2 strains also showed low dN/dS ratio $(0 \cdot 03-0 \cdot 16)$ suggesting purifying selection (Table $2 b)$. The first dataset consisted of sequences of all genotypes of DENV-2 $(n=73)$. The SLAC analysis revealed a dN/dS ratio of 0.036 and 67 negatively selected sites. The FEL analysis revealed 71 negatively selected sites using the HKY85 model of nucleotide substitution. Two weak positively selected sites (codon numbers 15 and 138) were observed by REL analysis. The second dataset comprised of only the sequences of the Cosmopolitan genotype $(n=42)$. These sequences showed a $\mathrm{dN} / \mathrm{dS}$ ratio of 0.074 and 17 negatively selected sites.

\section{DISCUSSION}

Dengue fever has emerged as a major health problem in India. Delhi, in the northern part of India, has become hyperendemic for dengue virus as all the four serotypes have been detected in Delhi. Numerous dengue fever outbreaks have been reported from Delhi including 1967, 1970, 1982, 1988, 1996, 2003, 2006, 2010 and 2013 [2, 11-18]. The samples in the present study were collected from the local health centre of Jamia Millia Islamia University in South East Delhi on the Yamuna river bank. Therfore, high humidity along with high temperature during the post-monsoon season favour mosquito breeding in this region. In addition, high population density and rapid urbanization also contribute to regular outbreaks of dengue fever in this region of Delhi [7]. An outbreak of dengue fever occurred in south-eastern region of Delhi during the post-monsoon season in 2014.

Our work provides an overview of this dengue fever outbreak in Delhi, 2014. Dengue virus was detected in $80 \%$ of the samples collected during this outbreak by 


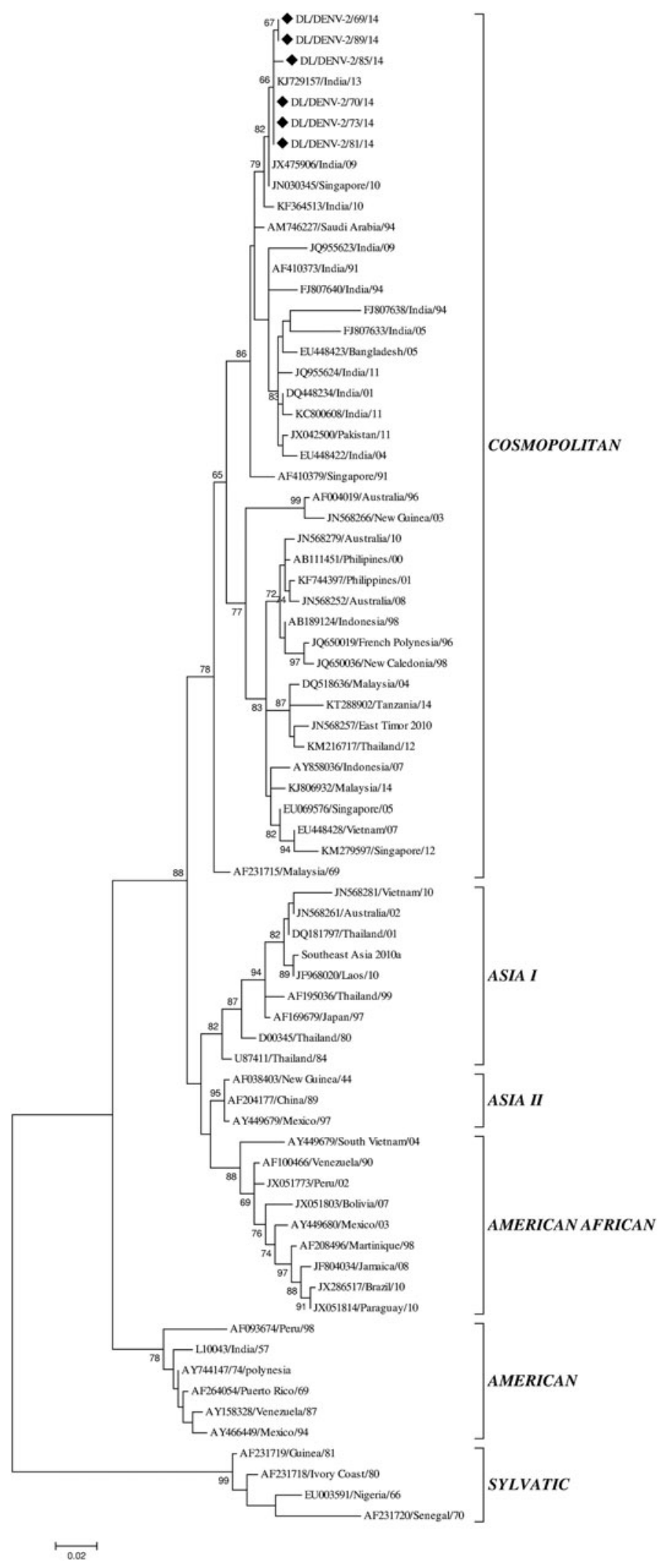

Fig. 2. Maximum-likelihood phylogenetic tree of DENV-2 strains. The list of 73 nucleotide sequences that were used to construct the tree is given in Supplementary Table S2b. The study sequences are indicated by a diamond symbol $(\boldsymbol{)})$. Bootstrap values are represented by the numbers on nodes generated by 1000 replications. Values $>65 \%$ are shown in the tree. 
Table 2. Selection pressure analysis of (a) DENV-1 and (b) DENV-2 of the envelope protein gene using the SLAC, FEL and REL methods

\begin{tabular}{|c|c|c|c|c|c|c|}
\hline \multirow{3}{*}{$\begin{array}{l}\text { (a) DENV-1 } \\
\text { Nucleotide substitution method }\end{array}$} & \multicolumn{4}{|c|}{ All genotypes } & \multicolumn{2}{|c|}{ American African genotype } \\
\hline & $\begin{array}{l}\text { SLAC } \\
(P=0 \cdot 05)\end{array}$ & $\begin{array}{l}\text { FEL } \\
(P=0 \cdot 1)\end{array}$ & $\begin{array}{l}\text { REL } \\
\text { (Bayes }\end{array}$ & value $=50)$ & $\begin{array}{l}\text { SLAC } \\
(P=0 \cdot 05)\end{array}$ & $\begin{array}{l}\text { FEL } \\
(P=0 \cdot 1)\end{array}$ \\
\hline & $\mathrm{dN} / \mathrm{dS}$ & $\begin{array}{l}\text { Positively selected } \\
\text { sites }\end{array}$ & $\mathrm{dN} / \mathrm{dS}$ & $\begin{array}{l}\text { Positively selected } \\
\text { sites }\end{array}$ & $\mathrm{dN} / \mathrm{dS}$ & $\begin{array}{l}\text { Positively selected } \\
\text { sites }\end{array}$ \\
\hline HKY85 & $0 \cdot 1056$ & $1(98)$ & $0 \cdot 5308$ & $2(56,98)$ & $0 \cdot 3528$ & 0 \\
\hline F81 & $0 \cdot 085$ & $2(9,98)$ & $0 \cdot 3063$ & $3(9,56,98)$ & $0 \cdot 101$ & $2(9,98)$ \\
\hline \multirow[t]{2}{*}{ (a) DENV-2 } & \multicolumn{4}{|c|}{ All genotypes } & \multicolumn{2}{|c|}{ Cosmopolitan } \\
\hline & $\begin{array}{l}\text { SLAC } \\
(P=0 \cdot 05)\end{array}$ & $\begin{array}{l}\text { FEL } \\
(P=0 \cdot 05)\end{array}$ & $\begin{array}{l}\text { REL } \\
\text { (Bayes }\end{array}$ & value $=50)$ & $\begin{array}{l}\text { SLAC } \\
(P=0 \cdot 05)\end{array}$ & $\begin{array}{l}\text { FEL } \\
(P=0 \cdot 05)\end{array}$ \\
\hline Nucleotide substitution method & $\mathrm{dN} / \mathrm{dS}$ & $\begin{array}{l}\text { Positively selected } \\
\text { sites }\end{array}$ & $\mathrm{dN} / \mathrm{dS}$ & $\begin{array}{l}\text { Positively selected } \\
\text { sites }\end{array}$ & $\mathrm{dN} / \mathrm{dS}$ & $\begin{array}{l}\text { Positively selected } \\
\text { sites }\end{array}$ \\
\hline HKY85 & $0 \cdot 036$ & 0 & & $2(15,138)$ & $0 \cdot 074$ & 0 \\
\hline F81 & 0.032 & 0 & - & & $0 \cdot 169$ & 0 \\
\hline
\end{tabular}

SLAC, Single likelihood ancestor counting; FEL, fixed effects likelihood; REL, random effects likelihood.

RT-PCR. Our earlier studies identified dengue virus in this region in $49 \%$ and $71 \%$ of samples during the outbreaks of 2011 and 2013, respectively [2, 7]. An earlier investigation from Delhi identified dengue virus in $69 \%$ of the samples by RT-PCR [3]. Das and colleagues detected dengue virus in $54 \%$ of samples from Orissa [19], and 49\% each from Kerala [6] and Madhya Pradesh [20]. Another investigation from Brazil reported a high percentage of dengue viral infection by PCR in 55\% of symptomatic patients [21]. DENV-1 (77\%) was the predominant serotype followed by DENV-2 in the present investigation. Phylogenetic analysis clustered the study strains with other recently reported sequences from India and adjoining countries. The DENV-1 study sequences formed a separate cluster within the American African genotype with sequences from India [2, 22], Singapore and Pakistan (I. Hussain et al., GenBank, unpublished data). The DENV-2 study sequences formed a cluster within the Cosmopolitan genotype along with strains from India and Singapore [23, 24]. These two genotypes have already been reported from India [1, 2, 7, 25].

One of the marked features of this investigation was the high percentage $(43 \%)$ of concurrent infection. The co-infected patients showed mild disease except three severe cases with no fatality. These three coinfected patients had mild haemorrhagic tendencies with one patient having a low platelet count. At the same time it is not possible to comment on the correlation of concurrent infection with disease severity due to limited samples size. However, future elaborate investigations on larger patient groups will determine the effect of concurrent infection on disease severity. However, it would be interesting to analyse the different circumstances that might contribute towards the understanding of concurrent infections. It has been suggested that concurrent infections with multiple serotypes of dengue virus occur due to co-circulation of these serotypes in the hyperendemic regions. Earlier studies have suggested that concurrent infections with multiple stereotypes along with their genotypes may affect the clinical presentation of the disease [3]. Moreover, it has been suggested that recombination in serotypes may occur in concurrent infections which might influence the emergence of more pathogenic and virulent strains [26]. Furthermore, earlier studies have indicated that dual infection with more than one serotype can occur in the vector probably due to multiple bloodmeals of the mosquitoes during a gonotrophic cycle [27, 28]. Further, high vector density in the endemic region may also contribute to the concurrent infections [6]. Nevertheless, additional investigations will determine the role of different parameters on concurrent infection with dengue virus serotypes. 


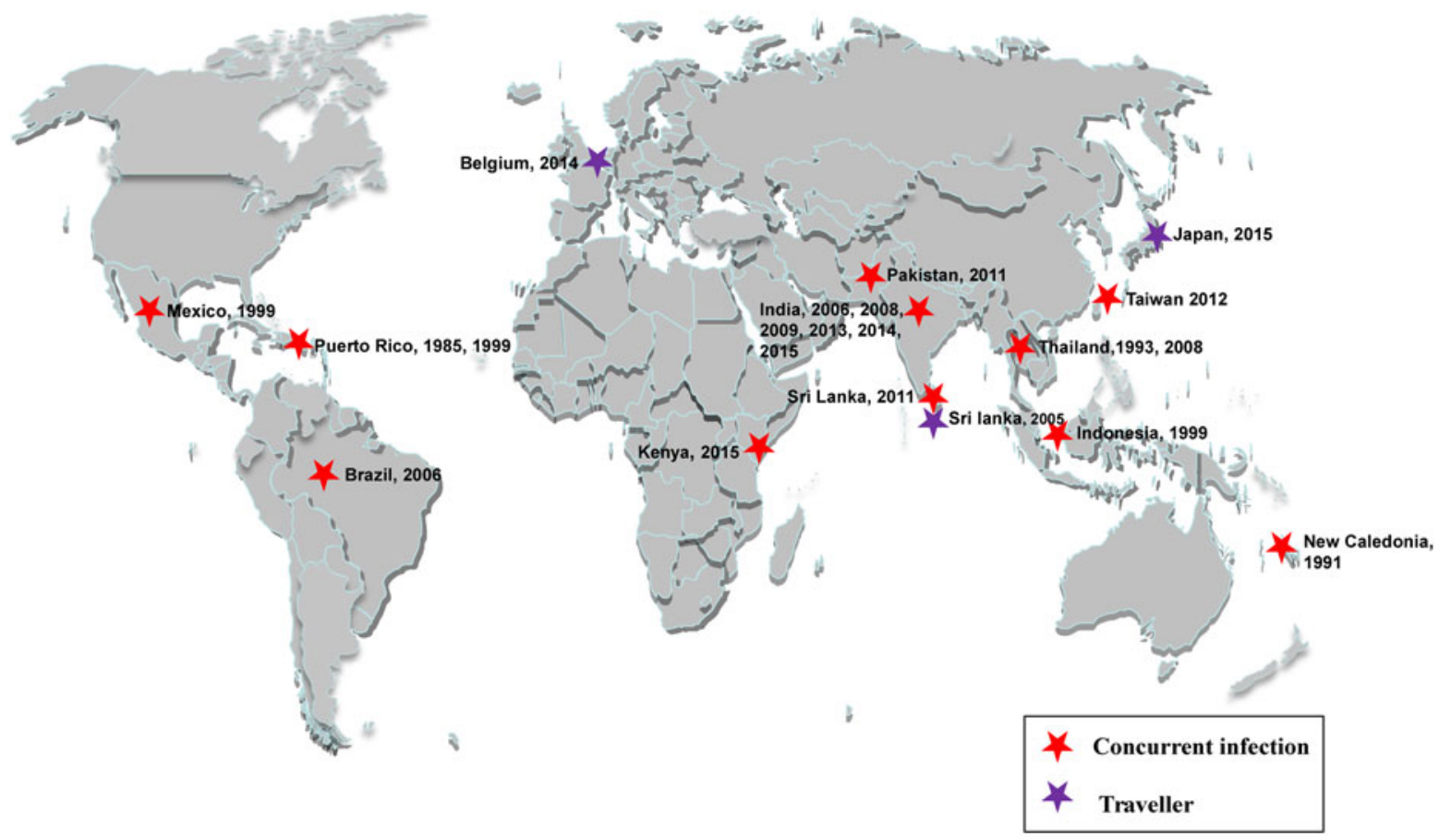

Fig. 3. World map showing concurrent infections with dengue virus serotypes in different geographical regions. The hotspot regions are indicated by a star symbol $(\star)$ followed by country and year of detection of the concurrent infection. The freely available world map was downloaded from the presentationmagazine.com website (http://www. presentationmagazine.com/world-maps-vector-editable-507.htm). The map was created and edited in PowerPoint.

Identification of the first case of concurrent infection with two dengue serotypes was reported in 1982 from Puerto Rico [29]. Other investigations from different geographical regions have reported concurrent infections in a significant number of samples from Singapore (17\%) [30], Sri Lanka (15\%), Indonesia (11\%), Taiwan (9.5\%) [22], Mexico, Puerto Rico and Indonesia together (5.5\%) [31] (Fig. 3). Concurrent infection was also reported from New Caledonia [32], Thailand [33, 34], and Pakistan [35]. Concurrent infections have also been reported in travellers from different geographical regions $[36,37]$. Interestingly, co-infection with more than one serotype of dengue virus has been reported in several studies from India (Fig. 4). Concurrent infection with the two different serotypes, DENV-1 and DENV-2, was identified in $43 \%$ of the samples in the present study. Similarly $43 \%$ co-infection was reported from Davangere, Karnataka [5] and 57\% from Thiruvananthapuram, Kerala [6], 32.5\% from Moreh, Manipur [38] and $19 \%$ from Delhi [3]. Our earlier work reported 9\% co-infections during 2011 [7], 14\% during 2013 [2] and $18 \%$ during 4 years of study from 2011-14 [39] in Delhi. Dual infection was also reported in other investigations from Delhi [4, 40, 41]. Thus, although perusal of the literature shows concurrent infections in several parts of the Indian subcontinent, the true disease burden and disease severity in concurrent infections remains unknown.

Dengue virus proteins have adapted to alternative hosts by selection of synonymous mutations leading to purifying selection. However, the envelope protein of the dengue virus is under immune selection pressure and thus prone to mutations. The selection pressure analysis of the envelope protein of DENV-1 and DENV-2 strains revealed a strong purifying selection. Similarly, purifying selection in the $\mathrm{E}$ protein has been reported previously in various investigations [19, 42, 43]. However, we were able to detect one codon in the American African genotype of DENV-1 viruses under weak positive selection pressure. Weak positive selection pressure in codons of the $\mathrm{E}$ protein have been reported previously [42]. Further detailed mutagenesis studies on this amino-acid change will define its role in the pathogenesis of dengue viral infection. The $\mathrm{E}$ protein of dengue virus is involved in attachment and fusion of the virion with the host cell. Domain III of the E protein was analysed, which is the putative receptor binding region. We identified a number of amino-acid mutations in this region. These mutations may alter the local secondary structure of the E protein which might affect the structure-function relationship [44]. One such 


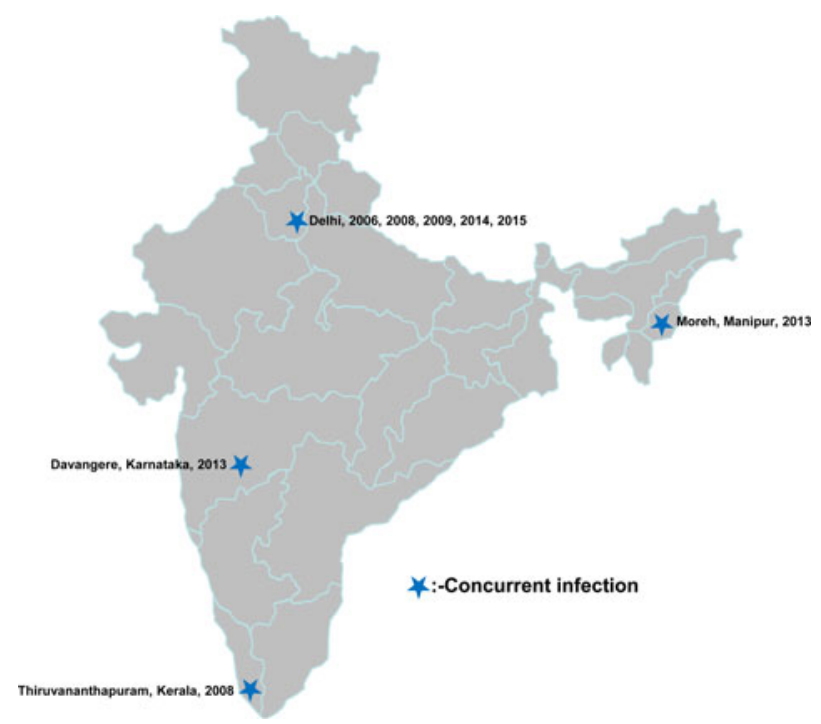

Fig. 4. Map of India showing concurrent infections with dengue virus serotypes. The hotspot regions are indicated by a star symbol $(\star)$ followed by city and year of detection of the concurrent infection. The map was downloaded from the presentationmagazine.com website (http://www.presentationmagazine.com/world-maps-vectoreditable-507.htm). The map was created and edited in PowerPoint.

recently reported mutation (T404I) was identified in two study DENV-2 strains. This mutation may affect low $\mathrm{pH}$-induced fusion of the virion. In addition, this mutation was also mapped to three different predicted T-cell epitopes that may affect the immunogenicity of the virus [44]. Further detailed investigations on these mutations will define there role in viral life cycle.

We are acutely aware of the limited sample size and confined infection areas. It would be of relevance to find the target tissues/cells of these viruses, the number and type of genes involved/affected and their possible up-/down-regulation during the course of concurrent infection. Establishing a genetic basis and genotypic delineation in the context of infection caused by a specific serotype would uncover the likely mechanism that has remained hitherto undiscovered. Titrating the viruses from across the affected individuals and correlating with diseased severity in the context of ethnicity would be yet another dimension to augment the epidemiology and aetiology of this disease. Manifestation of concurrent infection provides compelling evidence that the two serotypes of dengue virus are probably following the path of co-evolution despite having antigenic variabilities. Clearly, additional work along this line is envisaged to provide intellectual enrichment on disease burden.

\section{SUPPLEMENTARY MATERIAL}

For supplementary material accompanying this paper visit http://dx.doi.org/10.1017/S0950268816001990.

\section{ACKNOWLEDGEMENTS}

A. Tazeen is supported by Research Fellowship of the University Grants Commission, Government of India. S. Ali acknowledges the award of J.C. Bose National Fellowship from DST-SERB, New Delhi, India. This study was supported by King Saud University, Deanship of Scientific Research, College of Science Research Centre.

The study was funded by University Grants Commission, Government of India (http://www.ugc. ac.in) [grant no. 41-1287/2012 (SR)]. The funders had no role in study design, datacollection and analysis, decision to publish, or preparation of the manuscript.

\section{DECLARATION OF INTEREST}

None.

\section{REFERENCES}

1. Mishra G, et al. Molecular characterization of dengue viruses circulating during 2009-2012 in Uttar Pradesh, India. Journal of Medical Virology 2015; 87: 68-75.

2. Afreen N, et al. Molecular investigation of 2013 dengue fever outbreak from Delhi, India. PLoS Currents 2013; 6.

3. Bharaj $\mathbf{P}$, et al. Concurrent infections by all four dengue virus serotypes during an outbreak of dengue in 2006 in Delhi, India. Virology Journal 2008; 5: 1-5.

4. Gupta E, et al. The changing epidemiology of dengue in Delhi, India. Virology Journal 2006; 3: 8.

5. Vinodkumar C, et al. Episode of coexisting infections with multiple dengue virus serotypes in central Karnataka, India. Journal of Infection and Public Health 2013; 6: 302-306.

6. Anoop M, et al. Genetic characterization of dengue virus serotypes causing concurrent infection in an outbreak in Ernakulam, Kerala, South India. Indian Journal of Experimental Biology 2010; 48: 849-857.

7. Afreen $\mathbf{N}$, et al. Molecular characterization of dengue and chikungunya virus strains circulating in New Delhi, India. Microbiology and Immunology 2014; 58: 688-696.

8. Lanciotti RS, et al. Rapid detection and typing of dengue viruses from clinical samples by using reverse transcriptase-polymerase chain reaction. Journal of Clinical Microbiology 1992; 30: 545-551.

9. Wu W, et al. Molecular epidemiology of dengue viruses in southern China from 1978 to 2006. Virology Journal 2011; 8: 322.

10. Rodriguez-Roche R, et al. Dengue virus type 2 in Cuba, 1997: conservation of $\mathrm{E}$ gene sequence in isolates 
obtained at different times during the epidemic. Archives of Virology 2005; 150: 415-425.

11. Balaya $\mathbf{S}$, et al. Investigations on an outbreak of dengue in Delhi in 1967. Indian Journal of Medical Research 1969; 57: 767-774.

12. Diesh P, et al. An outbreak of dengue fever in Delhi 1970. Journal of Communicable Diseases 1972; 4: 8.

13. Rao C, et al. The 1982 epidemic of dengue fever in Delhi. Indian Journal of Medical Research 1985; 82: 271.

14. Kabra S, et al. Dengue haemorrhagic fever in children in Delhi. Bulletin of the World Health Organization 1992; 70: 105 .

15. Dar L, et al. The first major outbreak of dengue hemorrhagic fever in Delhi, India. Emerging Infectious Diseases 1999; 5: 589.

16. Singh N, et al. The 2003 outbreak of Dengue fever in Delhi, India. Southeast Asian Journal of Tropical Medicine and Public Health 2005; 36: 1174.

17. Pandey A, et al. The evolution of dengue over a decade in Delhi, India. Journal of Clinical Virology 2007; 40: 87-88.

18. Kumari R, et al. First dengue virus detection in Aedes albopictus from Delhi, India: its breeding ecology and role in dengue transmission. Tropical Medicine \& International Health 2011; 16: 949-954.

19. Das B, et al. Molecular investigations of dengue virus during outbreaks in Orissa state, Eastern India from 2010 to 2011. Infection, Genetics and Evolution 2013; 16: 401-410.

20. Barde $\mathbf{P}$, et al. Emergence of dengue in tribal villages of Mandla district, Madhya Pradesh, India. Indian Journal of Medical Research 2015; 141: 584.

21. Heinen LBdS, et al. Dengue outbreak in Mato Grosso state, midwestern Brazil. Revista do Instituto de Medicina Tropical de São Paulo 2015; 57: 489-496.

22. Huang $\mathbf{J}-\mathbf{H}$, et al. Molecular characterization and phylogenetic analysis of dengue viruses imported into Taiwan during 2008-2010. American Journal of Tropical Medicine and Hygiene 2012; 87: 349-358.

23. Sharma $\mathbf{P}$, et al. Continued circulation of DENV-2 (genotype IV) in Delhi, India. British Microbiology Research Journal 2015; 11: 1-8.

24. Lee K-S, et al. Dengue virus surveillance in Singapore reveals high viral diversity through multiple introductions and in situ evolution. Infection, Genetics and Evolution 2012; 12: 77-85.

25. Dash PK, et al. Complete genome sequencing and evolutionary phylogeography analysis of Indian isolates of Dengue virus type 1. Virus Research 2015; 195: 124-134.

26. Worobey M, et al. Widespread intra-serotype recombination in natural populations of dengue virus. Proceedings of the National Academy of Sciences USA 1999; 96: 7352-7357.

27. Thavara $\mathbf{U}$, et al. Double infection of heteroserotypes of dengue viruses in field populations of Aedes aegypti and Aedes albopictus (Diptera: Culicidae) and serological features of dengue viruses found in patients in southern Thailand Southeast Asian Journal of Tropical Medicine and Public Health 2006; 37: 468-476.

28. Scott TW, et al. A fitness advantage for Aedes aegypti and the viruses it transmits when females feed only on human blood. American Journal of Tropical Medicine and Hygiene 1997; 57: 235-239.

29. Gubler D, et al. A case of natural concurrent human infection with two dengue viruses. American Journal of Tropical Medicine and Hygiene 1985; 34: 170-173.

30. Wilder-Smith A, et al. Serological evidence for the cocirculation of multiple dengue virus serotypes in Singapore. Epidemiology and infection 2005; 133: 667-671.

31. Lorono-Pino M, et al. Common occurrence of concurrent infections by multiple dengue virus serotypes. American Journal of Tropical Medicine and Hygiene 1999; 61: 725-730.

32. Laille M, et al. Demonstration of concurrent dengue 1 and dengue 3 infection in six patients by the polymerase chain reaction. Journal of Medical Virology 1991; 34: $51-54$.

33. Maneekarn N, et al. Applications of polymerase chain reaction for identification of dengue viruses isolated from patient sera. Microbiology and Immunology 1993; 37: 41-47.

34. Chinnawirotpisan $\mathbf{P}$, et al. Detection of concurrent infection with multiple dengue virus serotypes in Thai children by ELISA and nested RT-PCR assay. Archives of Virology 2008; 153: 2225-2232.

35. Fatima Z, et al. Serotype and genotype analysis of dengue virus by sequencing followed by phylogenetic analysis using samples from three mini outbreaks - 2007 2009 in Pakistan. BMC Microbiology 2011; 11: 200.

36. Cnops L, et al. First dengue co-infection in a Belgian traveler returning from Thailand, July 2013. Journal of Clinical Virology 2014; 61: 597-599.

37. Moi ML, et al. Virological confirmation of concurrent dengue virus serotypes 1 and 4 by virus isolation using Fc-gamma receptor-expressing BHK cells. International Journal of Infectious Diseases 2015; 33: 177.

38. Khan S, et al. Dengue outbreak in an Indo-Myanmar boarder area: epidemiological aspects and risk factors. Tropical Biomedicine 2013; 30: 451-458.

39. Afreen $\mathbf{N}$, et al. Phylogenetic and molecular clock analysis of dengue serotype 1 and 3 from New Delhi, India. PLOS ONE 2015; 10: e0141628.

40. Gupta E, et al. Concurrent infection by two dengue virus serotypes among dengue patients. Indian Journal of Medical Microbiology 2008; 26: 402-403.

41. Chahar HS, et al. Co-infections with chikungunya virus and dengue virus in Delhi, India. Emerging infectious diseases 2009; 15: 1077.

42. Patil J, et al. Evolutionary dynamics of the American African genotype of dengue type 1 virus in India (1962-2005). Infection, Genetics and Evolution 2011; 11: 1443-1448.

43. Singh S, et al. Phylogenetic study reveals co-circulation of Asian II and cosmopolitan genotypes of dengue virus serotype 2 in Nepal during 2013. Infection, Genetics and Evolution 2015; 34: 402-409.

44. Afreen $\mathbf{N}$, et al. Evolutionary analysis of dengue serotype 2 viruses using phylogenetic and Bayesian methods from New Delhi, India. PLoS Neglected Tropical Diseases 2016; 10: e0004511. 